\title{
Formation of a Supramolecular Polymeric Adhesive via Water-Participant Hydrogen Bond Formation
}

Qiao Zhang, ${ }^{\mathrm{a} \dagger}$ Tao Li, ${ }^{\mathrm{a} \dagger}$ Abing Duan, ${ }^{\mathrm{b} \dagger}$ Shengyi Dong, ${ }^{\mathrm{a}^{*}}$ Wanxiang Zhao, ${ }^{\mathrm{a}^{*}}$ and Peter J. Stang ${ }^{\text {c* }^{*}}$

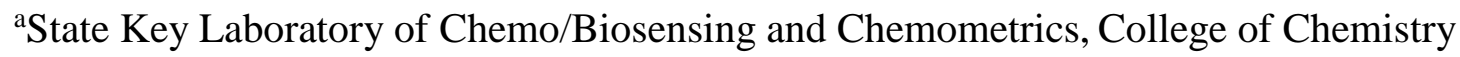
and Chemical Engineering, Hunan University, Changsha, Hunan 410082, P. R. China ${ }^{\mathrm{b}}$ College of Environment Science and Engineering, Hunan University, Changsha, Hunan 410082, P. R. China

${ }^{\mathrm{c}}$ Department of Chemistry, University of Utah, 315 South 1400 East, Room 2020, Salt Lake City, Utah 84112, United States

${ }^{\dagger}$ These authors contributed equally to this work

*E-mail:

dongsy@hnu.edu.cn

zhaowanxiang@hnu.edu.cn

stang@chem.utah.edu

\section{The PDF file includes:}

1. Materials and methods

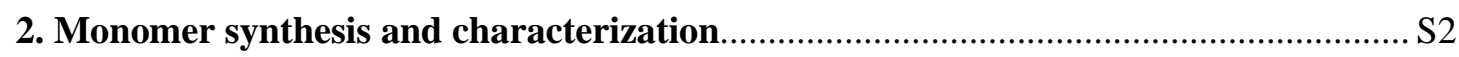

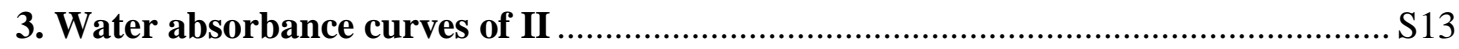

4. Powder X-Ray Diffraction (PXRD) pattern of II-water adhesive material ............... S14

5. Rheology measurements of II-water adhesive materials ......................................... S16

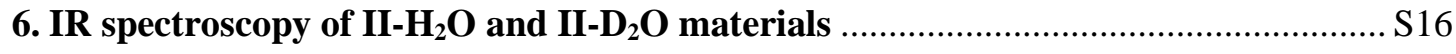

7. Thermogravimetric Analysis (TGA) characterization of II-water materials ........... S17

8. Scanning electron microscopy (SEM) of the micromorphology of II-water adhesive materials.

9. DFT calculation

10. References 


\section{Materials and methods}

All reagents were commercially available and used as supplied without further purification. BC7-NH2 and $\mathrm{Pt}\left(\mathrm{PEt}_{3}\right)_{2}(\mathrm{OTf})_{2}$ were prepared according to the reported methods. ${ }^{S 1,2}{ }^{1} \mathrm{H} /{ }^{31} \mathrm{P}$ NMR spectra were collected on a Varian Unity INOVA-400 or Bruker-AV400 with TMS as the internal standard. ${ }^{13} \mathrm{C}$ NMR spectra were recorded on a Bruker-AV400 spectrometer at $125 \mathrm{MHz}$. The electrospray-ionization Fouriertransform ion-cyclotron-resonance (ESI-FTICR) mass spectrometric experiments were performed with a Varian/IonSpec QFT-7 FTICR mass spectrometer equipped with a superconducting 7 Tesla magnet and a micromass Z-spray ESI ion source utilizing a stainless steel capillary with a $0.65 \mathrm{~mm}$ inner diameter. Fourier-transform IR (FT-IR) spectra were recorded on a Nicolet Avatar 320 FT-IR. Rheology measurements were done on an Anton Paar MCR 92. The laminator model PP15 was chosen with a diameter of $15 \mathrm{~mm}$ with the gap at $1 \mathrm{~mm}$. The adhesion strength measurements were performed on a pull-off adhesion tester (AT-A Automatic Adhesion Tester, PosiTest). Thermogravimetric analysis (TGA) was carried out using a TA Instruments Q5000IR analyzer with an automated vertical overhead thermobalance. The samples were heated at a rate of $10.0{ }^{\circ} \mathrm{C} / \mathrm{min}$. Scanning electron microscopy (SEM) experiments were performed on a high-resolution HITACHI SU 8030 scanning electron microscope. XRD data were collected in transmission mode on samples held on thin Mylar film in aluminum well plates on a Panalytical X'Pert PRO MPD equipped with a highthroughput screening XYZ stage, $\mathrm{x}$-ray focusing mirror, and PIXcel detector using Nifiltered $\mathrm{Cu} \mathrm{K} \alpha$ radiation. Data were measured over the range of $5^{\circ}$ to $60^{\circ}$ in $\sim 0.013^{\circ}$ steps over $60 \mathrm{~min}$. In IR, rheological tests and macroscopic tests, all samples contained about $1.3-1.5 \mathrm{wt} \%$ of water. 


\section{Monomer synthesis and characterization}
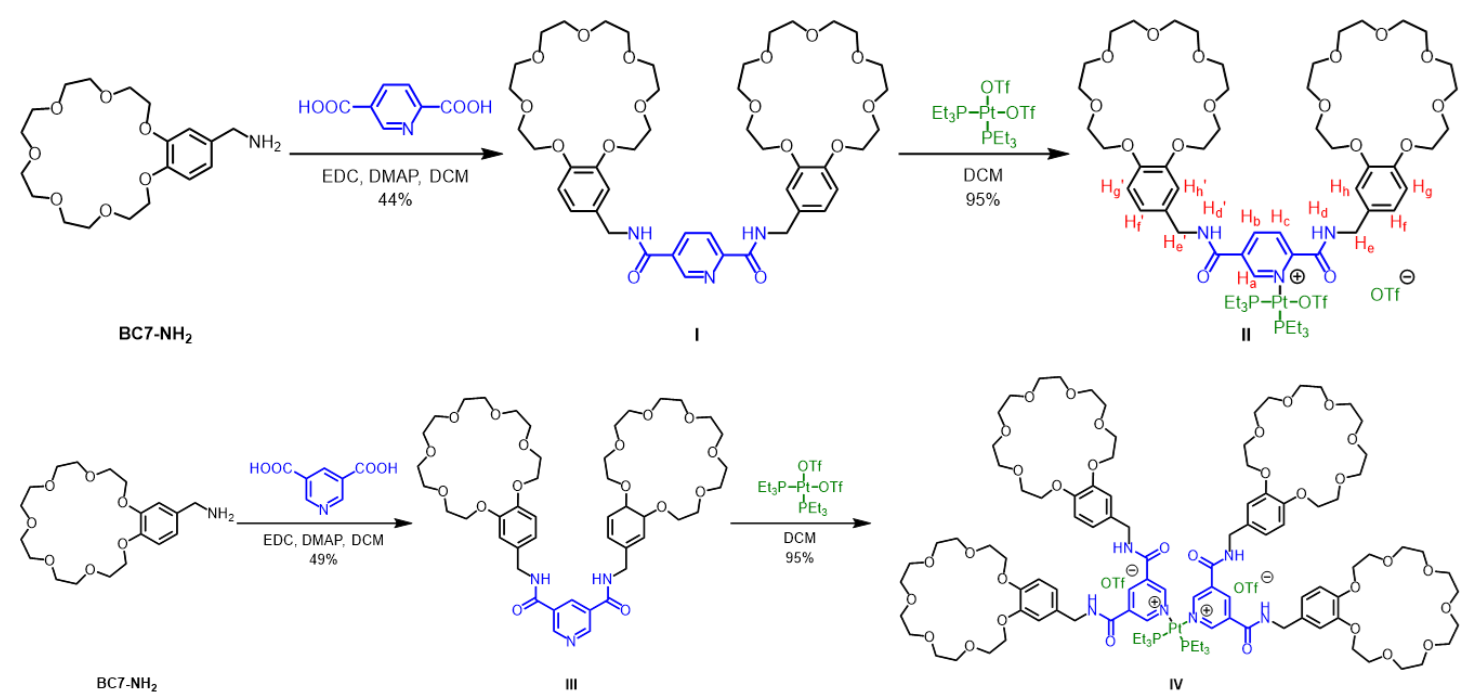

Scheme S1. Synthesis of I, II, III and IV.

Synthesis of I: EDC (4.62 g, 24.1 mmol), DMAP (0.100 g), BC7-NH2 (4.64 g, 12.1 mmol), pyridine-2,5-dicarboxylic acid (1.00 g, $6.03 \mathrm{mmol})$ and $\mathrm{CH}_{2} \mathrm{Cl}_{2}(50 \mathrm{~mL})$ were placed in a $100 \mathrm{~mL}$ flask. The mixture was stirred at room temperature overnight. Cold water $(20 \mathrm{~mL})$ was added to the reaction mixture and stirred at room temperature for 1 h. The organic layer was dried over anhydrous $\mathrm{MgSO}_{4}$ and concentrated in vacuo. After column chromatographic separation (silica gel, $\mathrm{CH}_{2} \mathrm{Cl}_{2}: \mathrm{MeOH}=30: 1, v: v$ ), $\mathbf{I}$ was obtained as a white solid (2.37 g, 44\%). Melting point: $144.1-146.3{ }^{\circ} \mathrm{C} . \mathbf{R}_{f}=0.25$ (silica gel, $\left.\mathrm{CH}_{2} \mathrm{Cl}_{2}: \mathrm{MeOH}=10: 1, v: v\right) .{ }^{1} \mathrm{H} \mathrm{NMR}\left(400 \mathrm{MHz}, \mathrm{CDCl}_{3}\right.$, room temperature) $\delta$ (ppm): 8.95(s, 1H), 8.33 (t, $J=8 \mathrm{~Hz}, 1 \mathrm{H}), 8.23(\mathrm{~d}, J=12 \mathrm{~Hz}, 1 \mathrm{H}), 8.21(\mathrm{~d}, J=8 \mathrm{~Hz}$, 1H) 7.01(s, 1H), 6.86-6.77 (m, 6H), $4.54(\mathrm{~d}, J=4 \mathrm{~Hz}, 4 \mathrm{H}), 4.11(\mathrm{~s}, 8 \mathrm{H}), 3.87(\mathrm{~s}, 8 \mathrm{H})$, $3.76(\mathrm{~s}, 8 \mathrm{H}), 3.70(\mathrm{~s}, 8 \mathrm{H}), 3.64-3.62(\mathrm{~m}, 16 \mathrm{H}) ;{ }^{13} \mathrm{C} \mathrm{NMR}\left(125 \mathrm{MHz}, \mathrm{CDCl}_{3}\right.$, room temperature) $\delta(\mathrm{ppm}): 164.77,163.38,151.70,149.11,148.53,148.43,147.22,136.20$, 132.03, 131.20, 130.85, 121.97, 121.07, 120.97, 114.36, 114.27, 114.18, 71.12, 71.09, 71.03, 70.55, 70.49, 69.74, 69.41, 69.36, 44.03, 43.33; HRESIMS: calcd. for $[\mathbf{I}+\mathrm{K}]^{+}$ (m/z): 940.3845; found 940.3864, error $2.0 \mathrm{ppm}$.

Synthesis of II: To $2 \mathrm{~mL}$ of $\mathrm{CH}_{2} \mathrm{Cl}_{2}$ were added I $(61.8 \mathrm{mg}, 0.0685 \mathrm{mmol})$ and $\mathrm{Pt}\left(\mathrm{PEt}_{3}\right)_{2}(\mathrm{OTf})_{2}(50.0 \mathrm{mg}, 0.0685 \mathrm{mmol})$. After stirring at room temperature for $10 \mathrm{~h}$, the solution was concentrated by flushing with $\mathrm{N}_{2}$ gas, followed by the addition of 5 $\mathrm{mL}$ diethyl ether. The resulting precipitate was collected by centrifugation to give II as 
a yellow solid (106 mg, 95\%). ${ }^{1} \mathrm{H} \mathrm{NMR}\left(400 \mathrm{MHz}, \mathrm{CDCl}_{3}\right.$, room temperature) $\delta(\mathrm{ppm})$ : $10.13(\mathrm{~s}, 1 \mathrm{H}), 9.16$ (s, 1H), 9.02 (d, $J=8 \mathrm{~Hz}, 1 \mathrm{H}), 8.86$ (d, $J=8 \mathrm{~Hz}, 1 \mathrm{H}), 8.63$ (s, 1H), $6.93(\mathrm{~s}, 1 \mathrm{H}), 6.86-6.77(\mathrm{~m}, 3 \mathrm{H}), 6.69(\mathrm{~d}, J=8 \mathrm{~Hz}, 2 \mathrm{H}), 4.67(\mathrm{~s}, 2 \mathrm{H}), 4.47$ (s, 2H), 4.00 $(\mathrm{s}, 8 \mathrm{H}), 3.76(\mathrm{~s}, 8 \mathrm{H}), 3.61-3.45(\mathrm{~m}, 32 \mathrm{H}), 2.13(\mathrm{~s}, 12 \mathrm{H}), 1.27-1.17(\mathrm{~m}, 18 \mathrm{H}) ;{ }^{31} \mathrm{P}\left\{{ }^{1} \mathrm{H}\right\}$ NMR (161.8 MHz, acetone- $d_{6}$, room temperature) $\delta(\mathrm{ppm}): 10.65\left(\mathrm{~s},{ }^{195} \mathrm{Pt}\right.$ satellites, $\left.{ }^{1} J_{\mathrm{Pt}-\mathrm{P}}=1719 \mathrm{~Hz}\right)$. HRESIMS calcd for [II-OTf-HOTf] ${ }^{+}(\mathrm{m} / \mathrm{z})$ : 1331.5600; found: 1331.5644, error $3.2 \mathrm{ppm}$.

Synthesis of III: EDC (1.92 g, 10.0 mmol), DMAP (0.10 g), BC7-NH2 (1.93 g, 5.0 mmol), pyridine-3,5-dicarboxylic acid $(0.42 \mathrm{~g}, 2.0 \mathrm{mmol})$ and $\mathrm{CH}_{2} \mathrm{Cl}_{2}(50 \mathrm{~mL})$ were placed in a $100 \mathrm{~mL}$ flask. Then the mixture was stirred at room temperature. Cold water $(20 \mathrm{~mL})$ was added to the reaction mixture and stirred at room temperature for $1 \mathrm{~h}$. The organic layer was dried over anhydrous $\mathrm{MgSO}_{4}$ and concentrated in vacuo. After column chromatographic separation (silica gel, $\mathrm{CH}_{2} \mathrm{Cl}_{2}: \mathrm{MeOH}=30: 1, v: v$ ), III was obtained as a yellow liquid (1.11 g, 49\%). $\mathbf{R}_{f}=0.25$ (silica gel, $\mathrm{CH}_{2} \mathrm{Cl}_{2}: \mathrm{MeOH}=10: 1$, $v: v) ;{ }^{1} \mathrm{H}$ NMR (400 MHz, $\mathrm{CDCl}_{3}$, room temperature) $\delta(\mathrm{ppm}): 9.10(\mathrm{~s}, 2 \mathrm{H}), 8.63(\mathrm{~s}, 1 \mathrm{H})$, 7.87(s, 2H), 6.82(s, 2H), 6.75 (d, $J=8 \mathrm{~Hz}, 2 \mathrm{H}), 6.67$ (d, $J=8 \mathrm{~Hz}, 2 \mathrm{H}), 4.40(\mathrm{~d}, J=8$ $\mathrm{Hz}, 4 \mathrm{H}), 4.05$ (s, 8H), 3.86-3.82 (m, 8H), 3.74-3.68 (m, 16H), 3.63-3.62 (m, $16 \mathrm{H}) ;{ }^{13} \mathrm{C}$ NMR (125 MHz, $\mathrm{CDCl}_{3}$, room temperature) $\delta$ (ppm): 164.93, 151.04, 148.88, 148.35, 133.26, 131.10, 129.41, 121.04, 114.52, 114.16, 71.06, 70.99, 70.92, 70.54, 70.43, 69.86, 69.80, 69.34, 49.16, 43.77; HRESIMS: $\mathrm{m} / \mathrm{s}$ calcd for $[\mathrm{M}+\mathrm{K}]^{+} \mathrm{C}_{45} \mathrm{H}_{63} \mathrm{KN}_{3} \mathrm{O}_{16}$, 940.3845; found 940.3890, error $4.8 \mathrm{ppm}$.

Synthesis of IV: To $2 \mathrm{~mL}$ of $\mathrm{CH}_{2} \mathrm{Cl}_{2}$ were added $\mathbf{I}(74.1 \mathrm{mg}, 0.0822 \mathrm{mmol})$ and $\mathrm{Pt}\left(\mathrm{PEt}_{3}\right)_{2}(\mathrm{OTf})_{2}(30.0 \mathrm{mg}, 0.0411 \mathrm{mmol})$. After stirring at room temperature for $10 \mathrm{~h}$, the solution was concentrated by flushing with $\mathrm{N}_{2}$ gas, followed by the addition of 5 $\mathrm{mL}$ diethyl ether. The resulting precipitate was collected by centrifugation to give II as a yellow solid (95.8 mg, 92\%). ${ }^{1} \mathrm{H} \mathrm{NMR}\left(400 \mathrm{MHz}, \mathrm{CDCl}_{3}\right.$, room temperature) $\delta(\mathrm{ppm})$ : $9.77(\mathrm{~s}, 3 \mathrm{H}), 9.17(\mathrm{~s}, 1 \mathrm{H}), 8.80(\mathrm{~s}, 2 \mathrm{H}), 8.64-8.56(\mathrm{~m}, 3 \mathrm{H}), 8.45(\mathrm{~s}, 1 \mathrm{H}), 7.04-7.00(\mathrm{~m}$, 4H), 6.91-6.89 (m, 8H), 4.54 (d, $J=8 \mathrm{~Hz}, 2 \mathrm{H}), 4.45$ (d, $J=8 \mathrm{~Hz}, 6 \mathrm{H}), 4.11(\mathrm{~s}, 16 \mathrm{H})$, $3.83(\mathrm{~s}, 16 \mathrm{H}), 3.68(\mathrm{~s}, 16 \mathrm{H}), 3.64(\mathrm{~s}, 16 \mathrm{H}), 3.58(\mathrm{~s}, 32 \mathrm{H}), 2.09-2.05(\mathrm{~m}, 12 \mathrm{H}), 1.37-$ $1.24(\mathrm{~m}, 18 \mathrm{H}) ;{ }^{31} \mathrm{P}\left\{{ }^{1} \mathrm{H}\right\} \mathrm{NMR}\left(161.8 \mathrm{MHz}\right.$, acetone- $d_{6}$, room temperature) $\delta(\mathrm{ppm})$ :

$-3.31\left(\mathrm{~s},{ }^{195} \mathrm{Pt}\right.$ satellites, $\left.{ }^{1} \boldsymbol{J}_{\mathrm{Pt}-\mathrm{P}}=532 \mathrm{~Hz}\right)$. HRESIMS calcd for $\left.[\mathbf{I V}-2 \mathrm{OTf}]\right]^{2+}(\mathrm{m} / \mathrm{z})$ : 
1117.4951; found: 1117.4917 , error: $3.0 \mathrm{ppm}$.

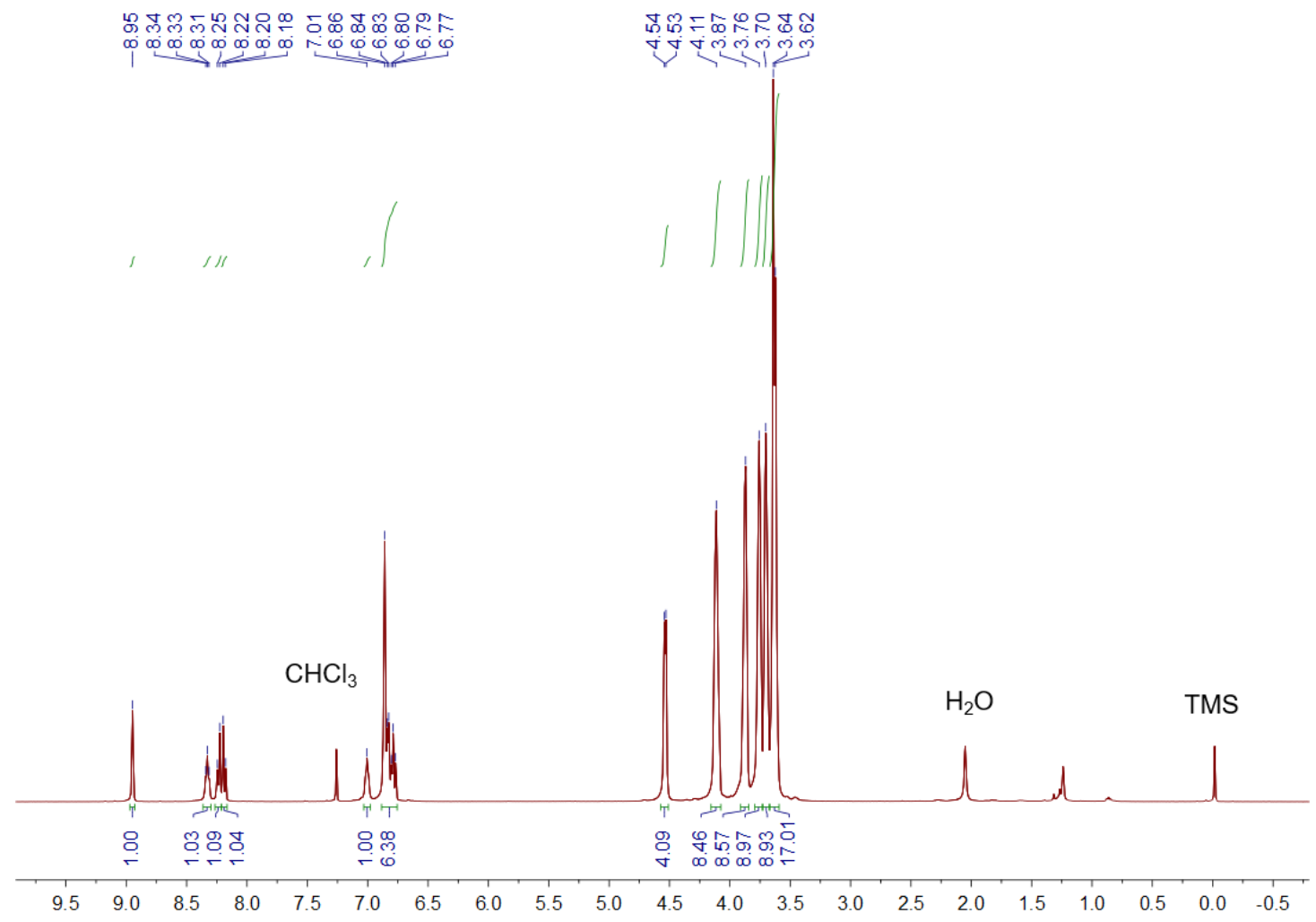

Figure S1. ${ }^{1} \mathrm{H}$ NMR spectrum (400 MHz, $\mathrm{CDCl}_{3}$, room temperature) of $\mathbf{I}$.

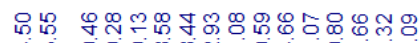

我它

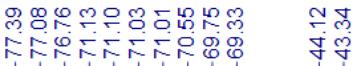

$\mathrm{CHCl}_{3}$

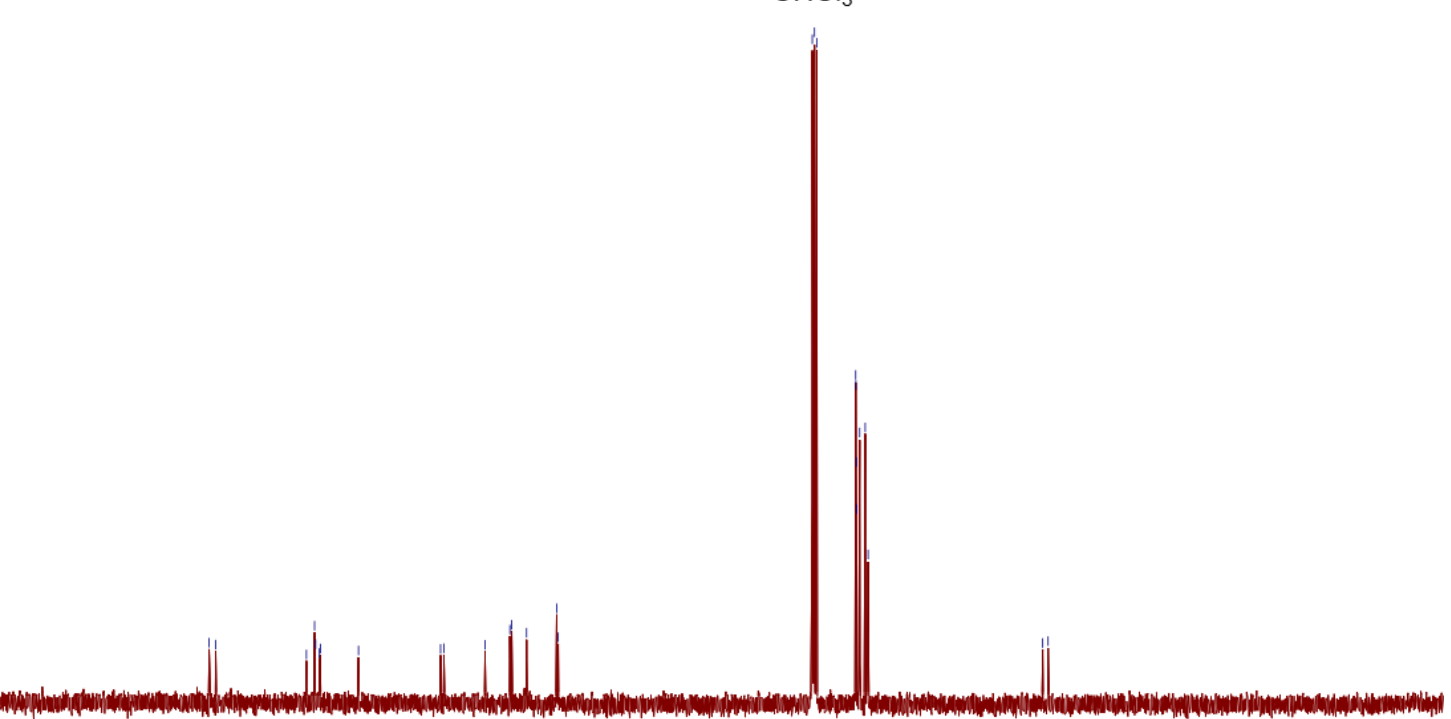

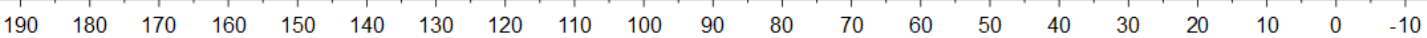

Figure S2. ${ }^{13} \mathrm{C}$ NMR spectrum (125 MHz, $\mathrm{CDCl}_{3}$, room temperature) of $\mathbf{I}$. 


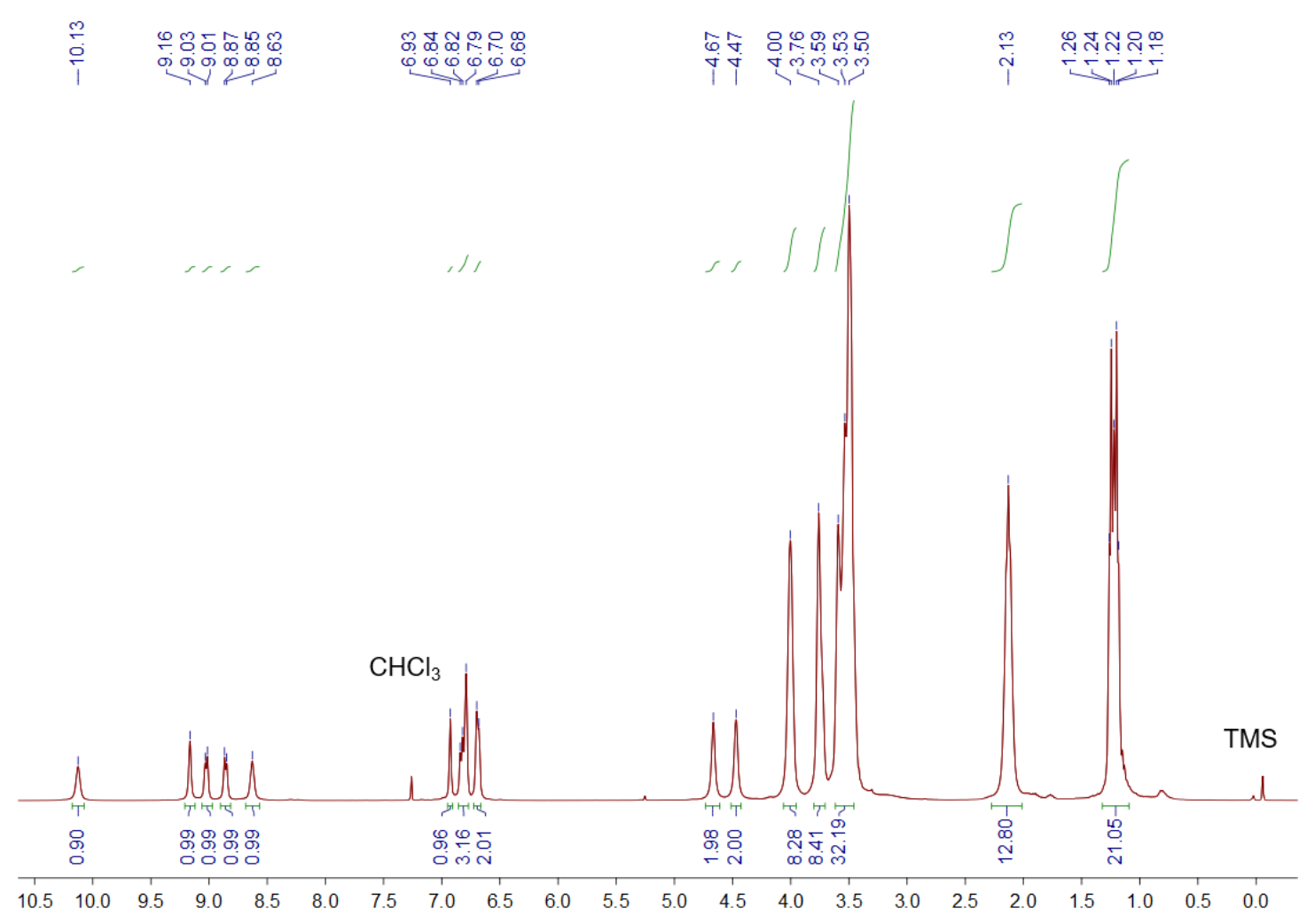

Figure S3. ${ }^{1} \mathrm{H}$ NMR spectrum $\left(400 \mathrm{MHz}, \mathrm{CDCl}_{3}\right.$, room temperature) of II.

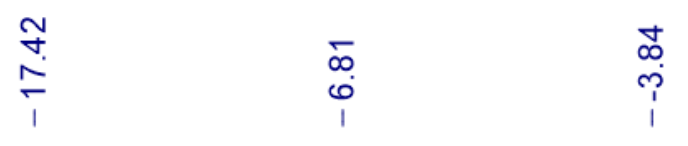

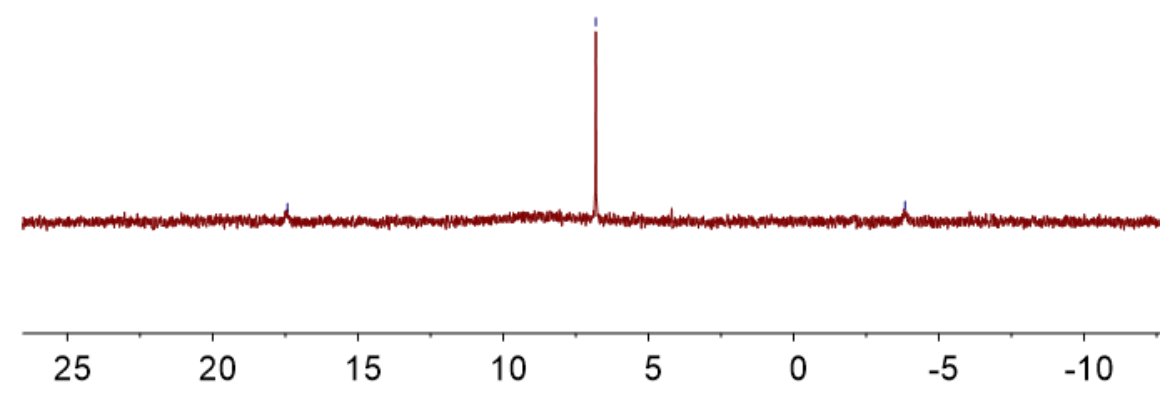

Figure S4. ${ }^{31} \mathrm{P}\left\{{ }^{1} \mathrm{H}\right\}$ NMR spectrum (400 MHz, acetone- $d_{6}$, room temperature) of II. 


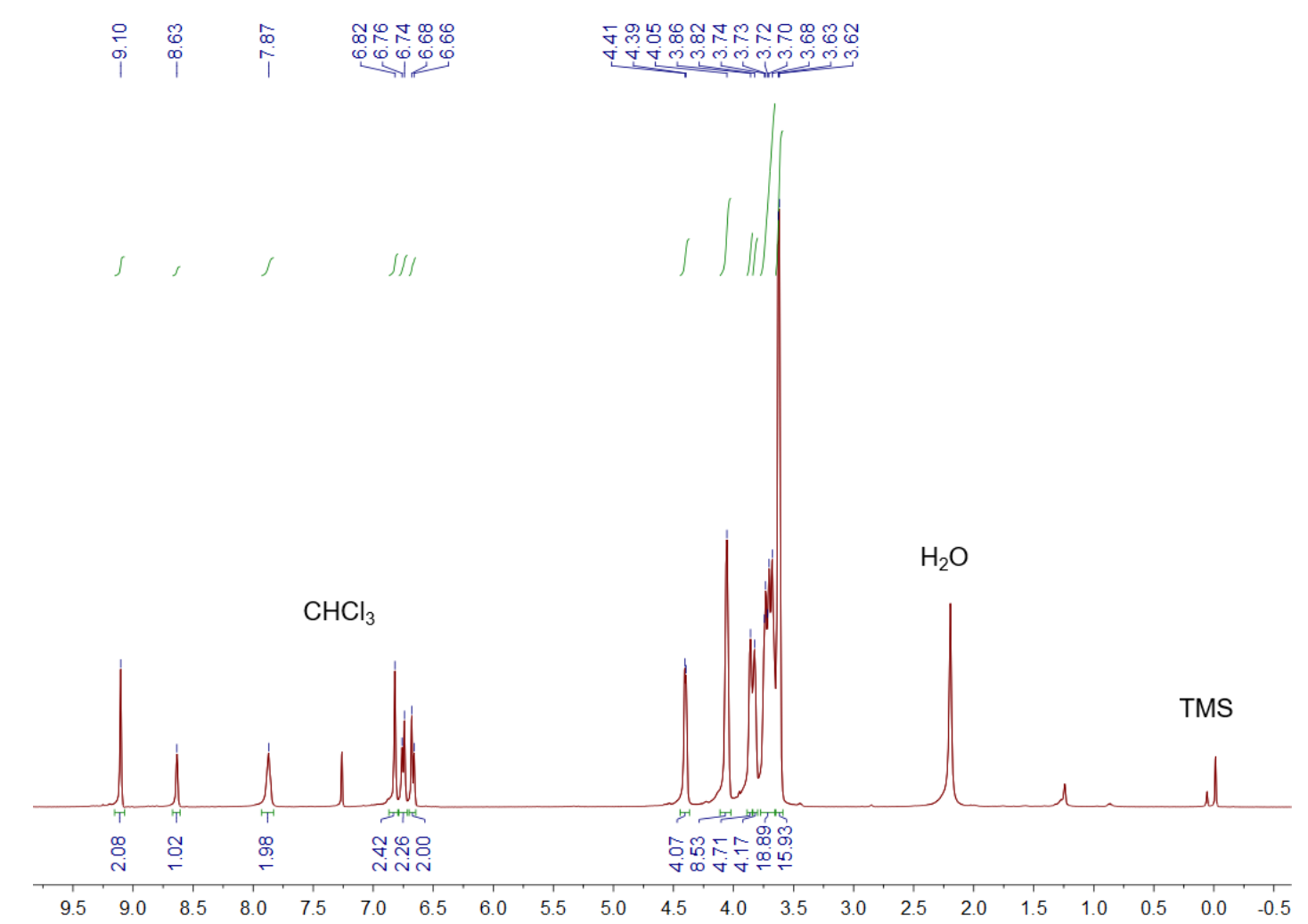

Figure S5. ${ }^{1} \mathrm{H}$ NMR spectrum (400 MHz, $\mathrm{CDCl}_{3}$, room temperature) of III.

\begin{tabular}{|c|c|c|}
\hline 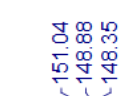 & 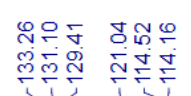 & 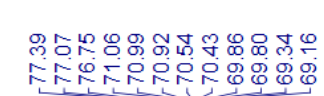 \\
\hline & & $\mathrm{CHCl}_{3}$ \\
\hline
\end{tabular}

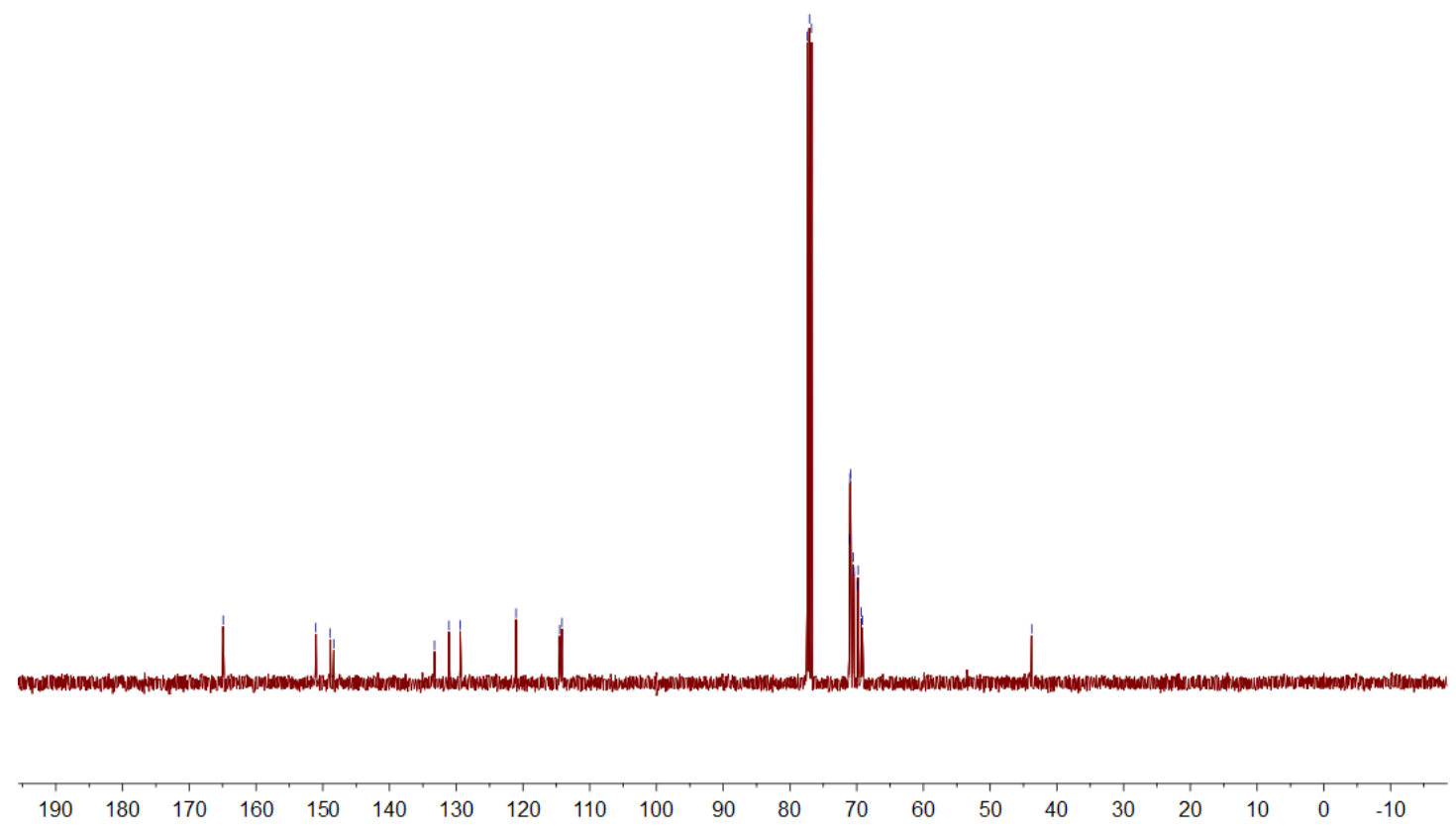

Figure S6. ${ }^{13} \mathrm{C}$ NMR spectrum $\left(125 \mathrm{MHz}, \mathrm{CDCl}_{3}\right.$, room temperature) of III. 


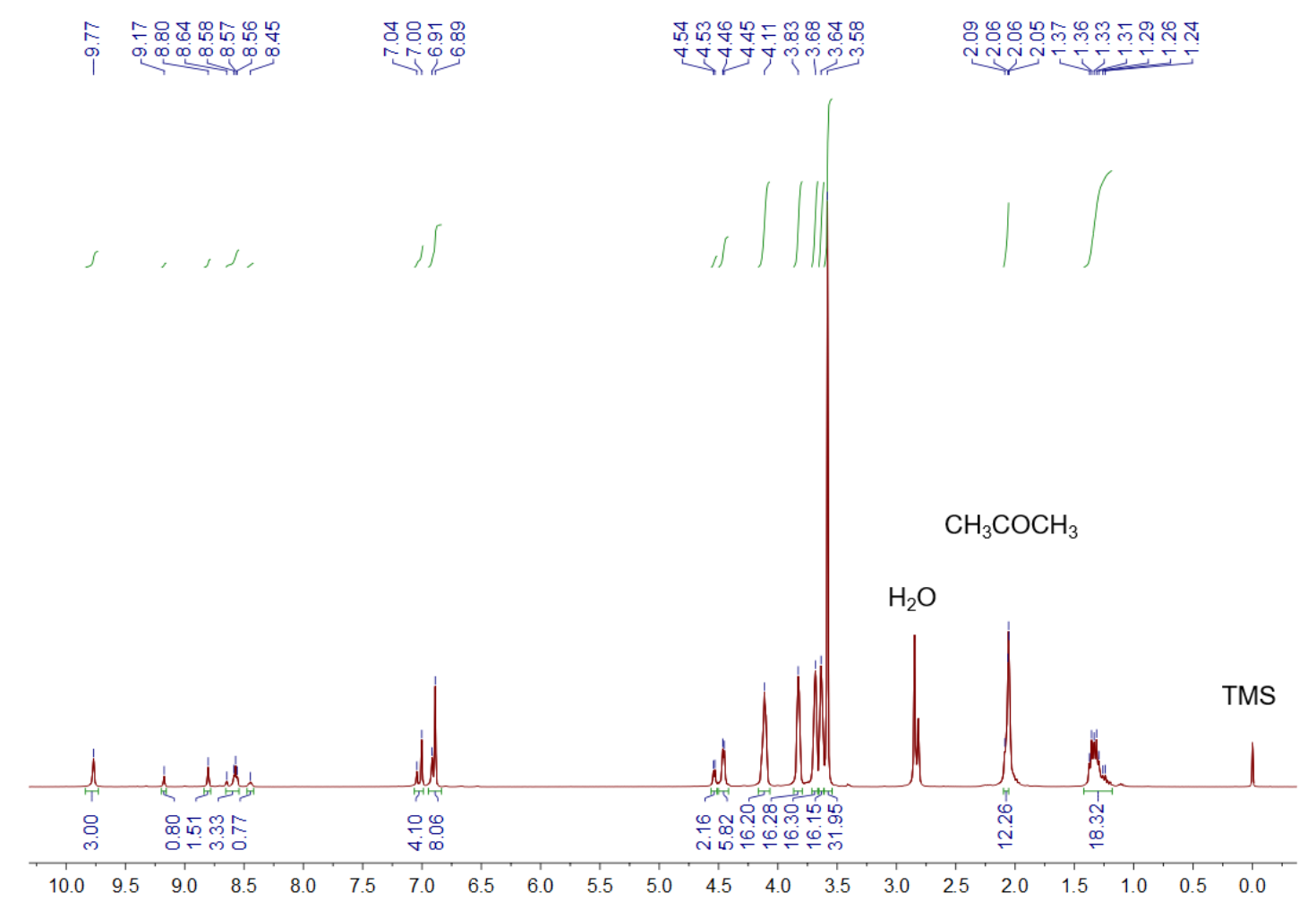

Figure S7. ${ }^{1} \mathrm{H}$ NMR spectrum (400 MHz, acetone- $d_{6}$, room temperature) of IV.
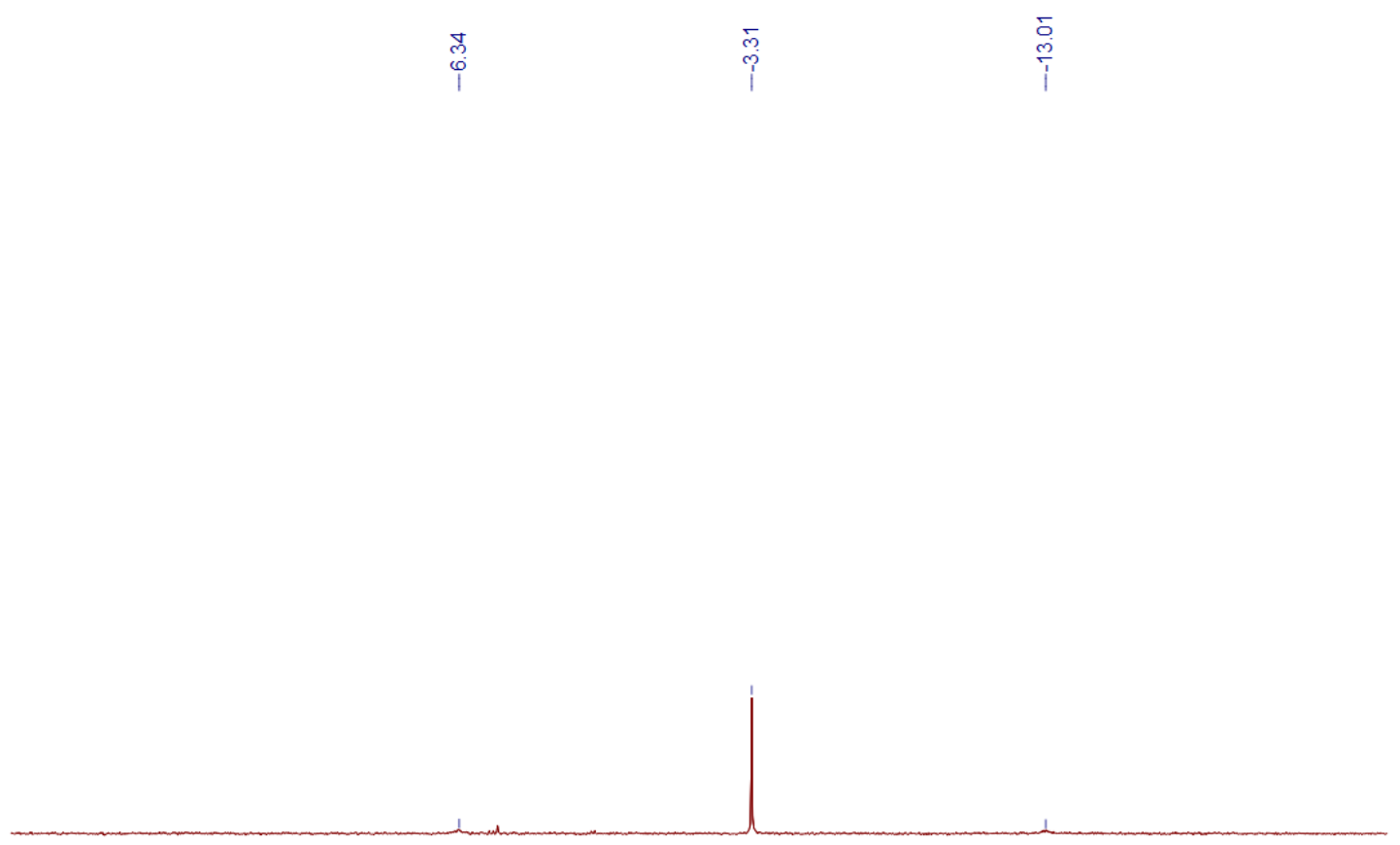

\begin{tabular}{llllllll}
1 & 1 & 1 & 1 & 1 & 1 & 1 \\
\hline 20 & 15 & 10 & 5 & 0 & -5 & -10 & -15
\end{tabular}

Figure S8. ${ }^{31} \mathrm{P}\left\{{ }^{1} \mathrm{H}\right\}$ NMR spectrum $\left(400 \mathrm{MHz}\right.$, acetone- $d_{6}$, room temperature) of IV. 

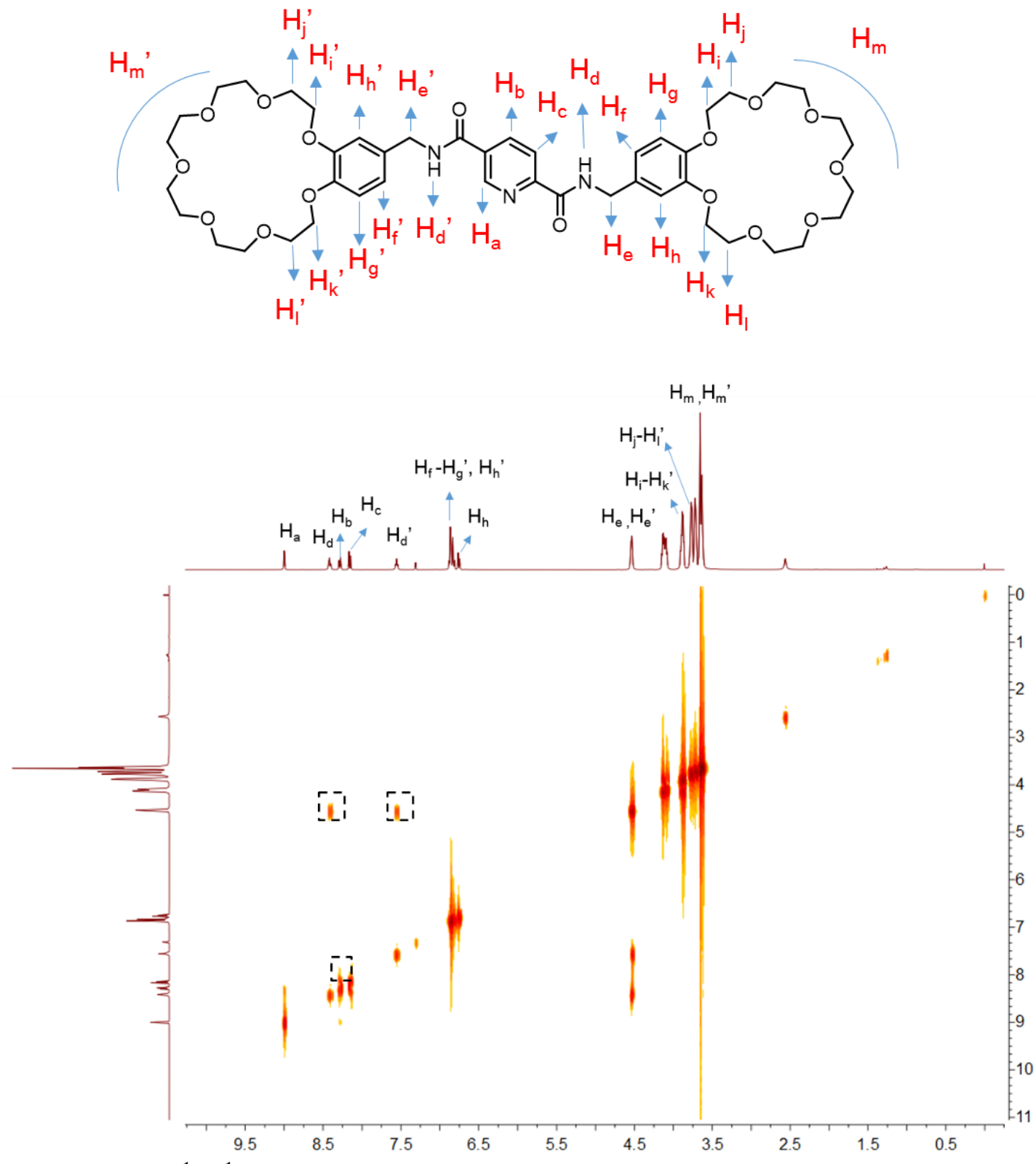

Figure S9. ${ }^{1} \mathrm{H}-{ }^{1} \mathrm{H}$ COSY spectrum of $\mathbf{I}\left(400 \mathrm{MHz}, \mathrm{CDCl}_{3}\right.$, room temperature). 

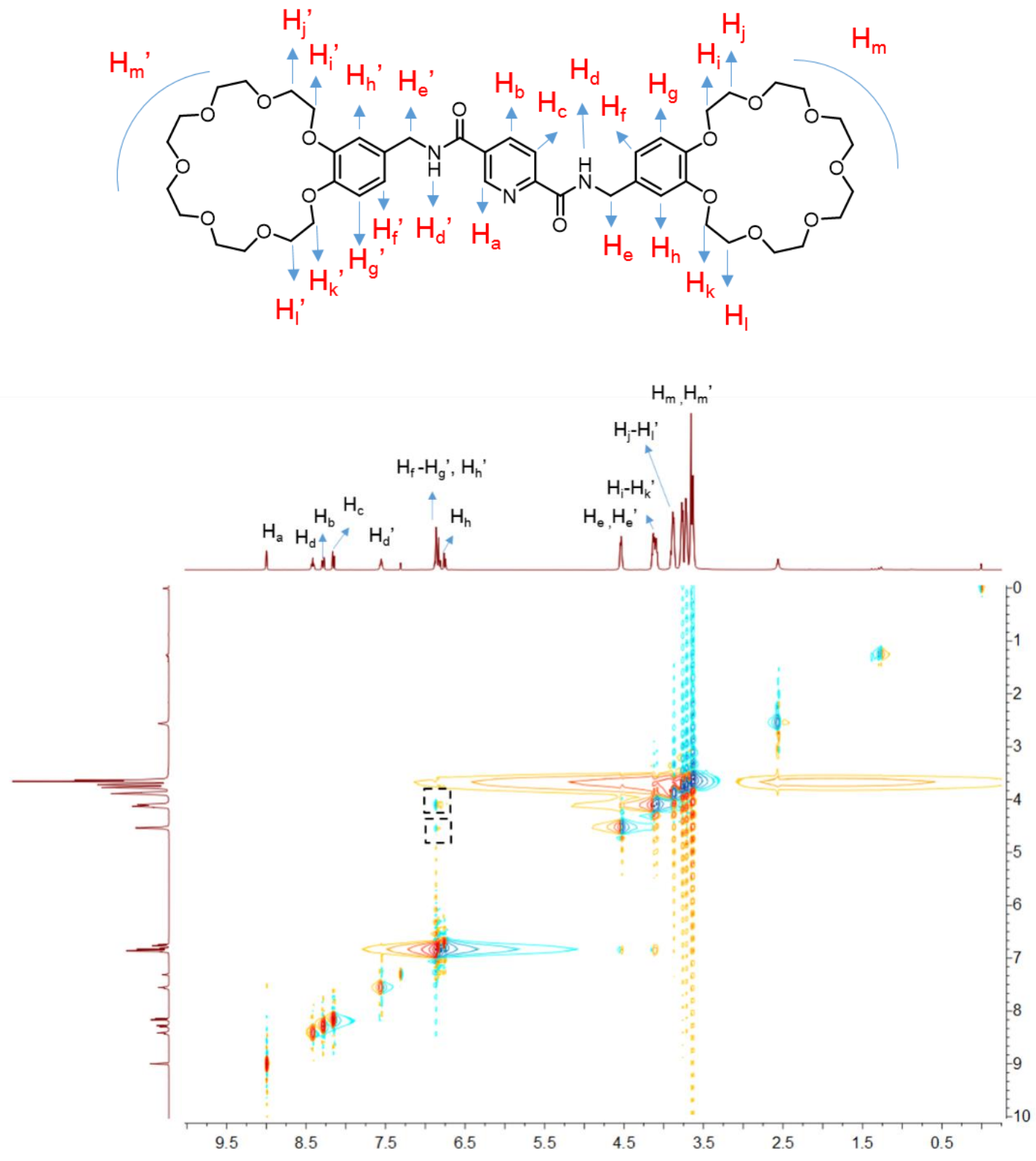

Figure S10. ${ }^{1} \mathrm{H}-{ }^{1} \mathrm{H}$ NOESY spectrum of $\mathbf{I}\left(400 \mathrm{MHz}, \mathrm{CDCl}_{3}\right.$, room temperature). 

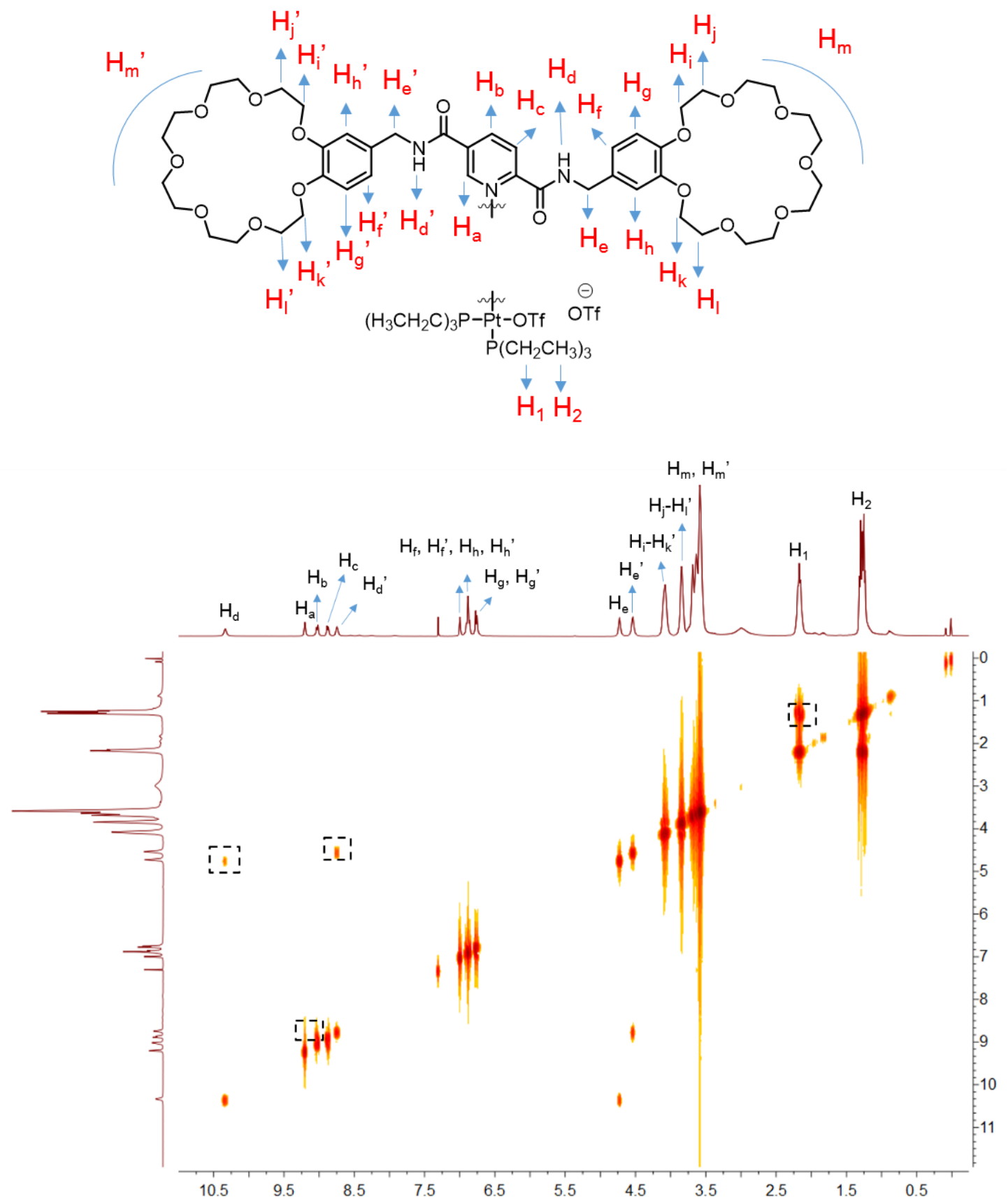

Figure S11. ${ }^{1} \mathrm{H}-{ }^{1} \mathrm{H}$ COSY spectrum of II (400 MHz, $\mathrm{CDCl}_{3}$, room temperature). 

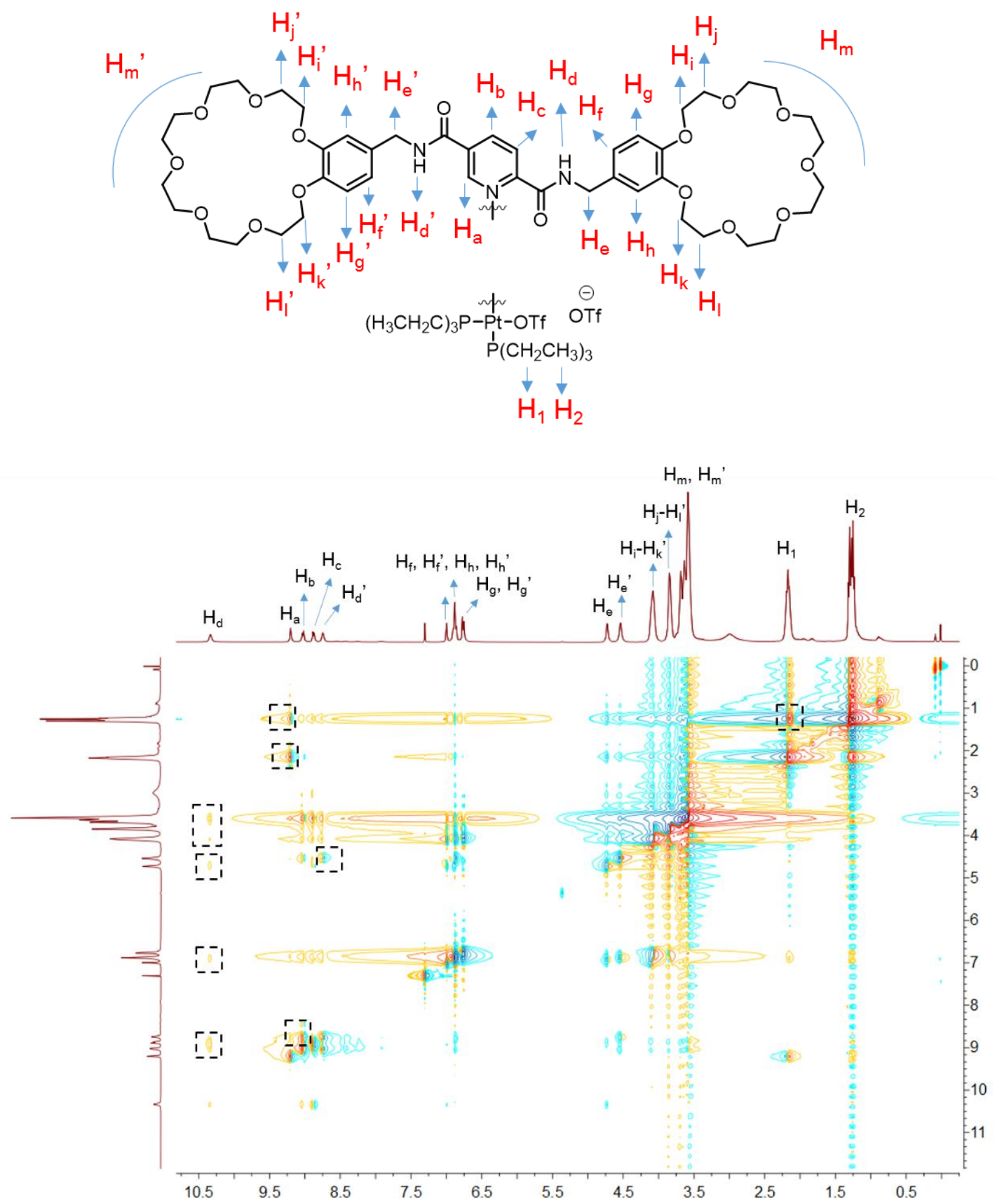

Figure S12. ${ }^{1} \mathrm{H}-{ }^{1} \mathrm{H}$ NOESY spectrum of II ( $400 \mathrm{MHz}, \mathrm{CDCl}_{3}$, room temperature). 

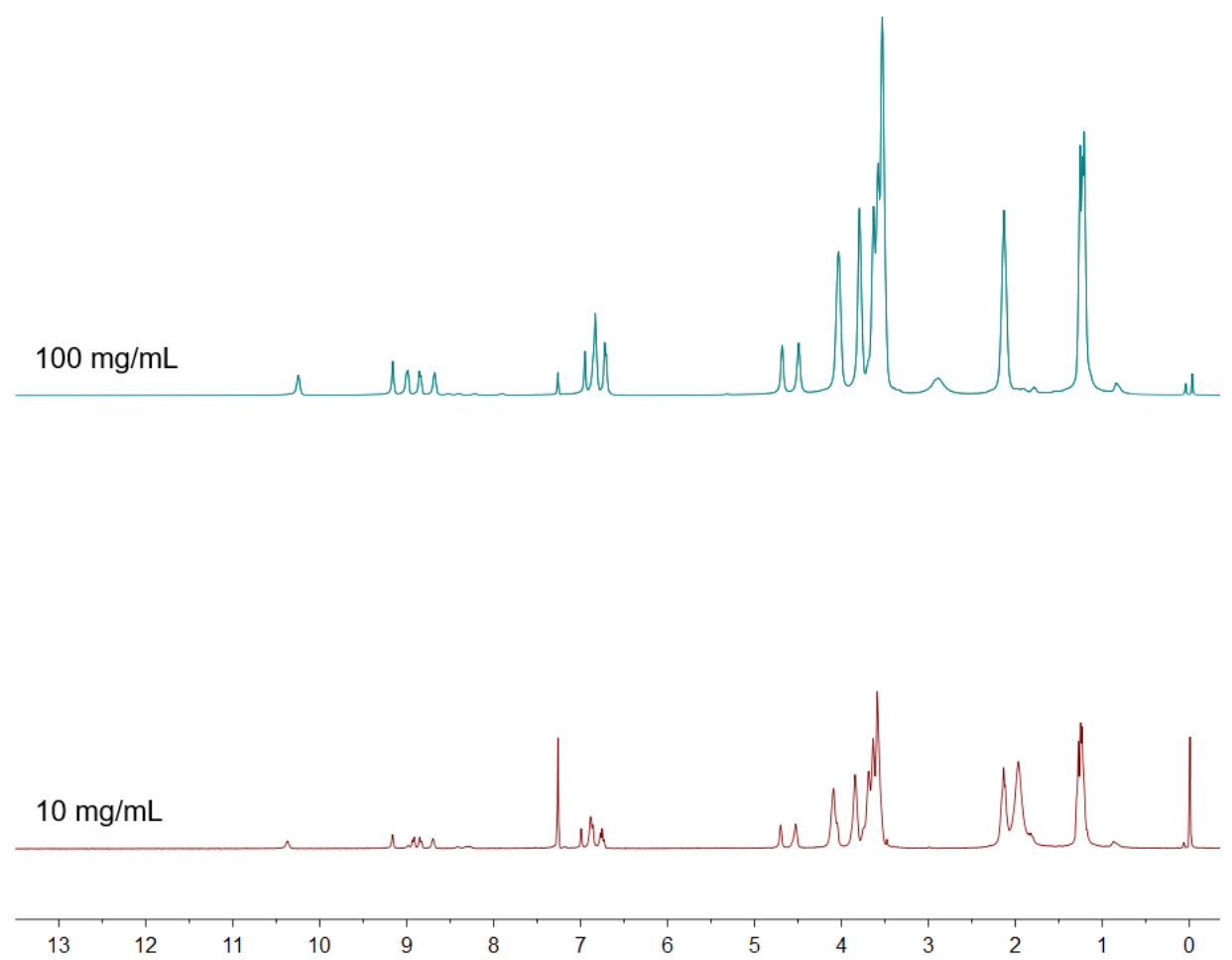

Figure S13. Concentration-dependent ${ }^{1} \mathrm{H}$ NMR spectra of II $\left(400 \mathrm{MHz}, \mathrm{CDCl}_{3}\right.$, room temperature).

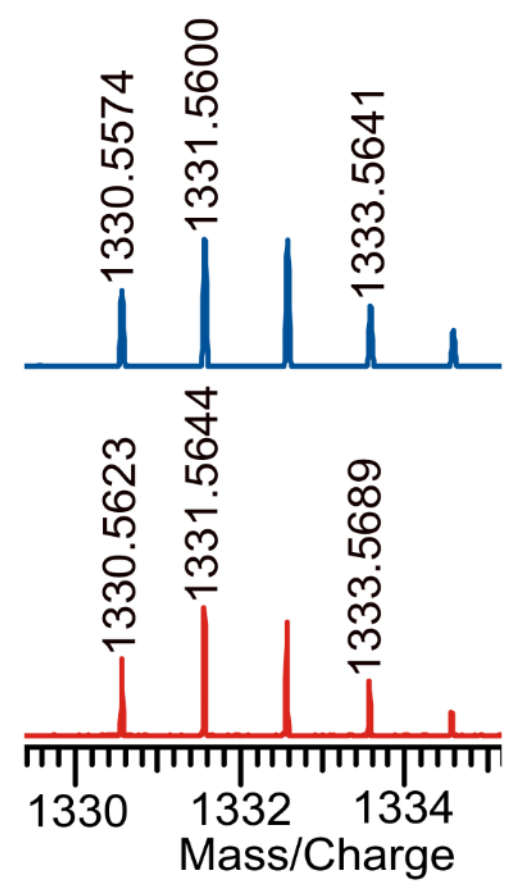

Figure S14. ESI-FTICR-MS experimental data (red) and predicted isotope distribution (blue) of [II-OTf-HOTf] ${ }^{+}$. 


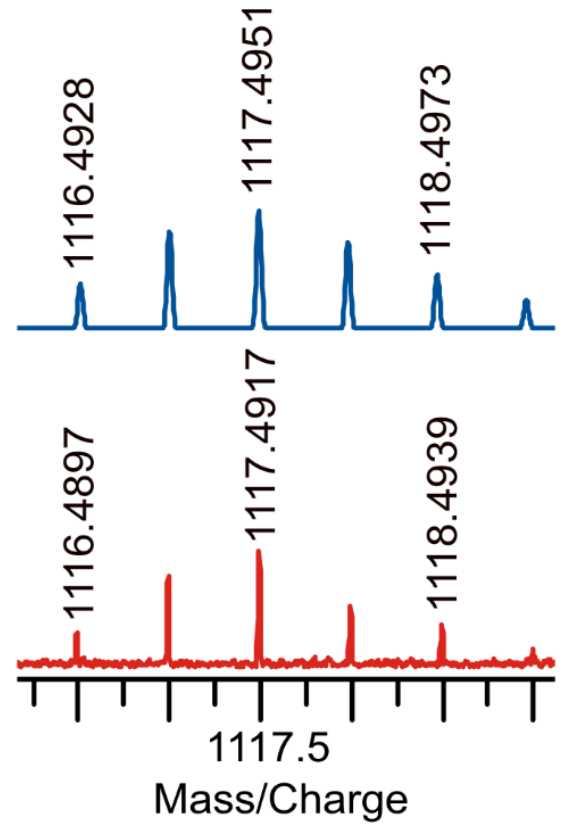

Figure S15. ESI-FTICR-MS experimental data (red) and predicted isotope distribution (blue) of [IV-2OTf $]^{2+}$.

\section{Water absorbance curves of II}

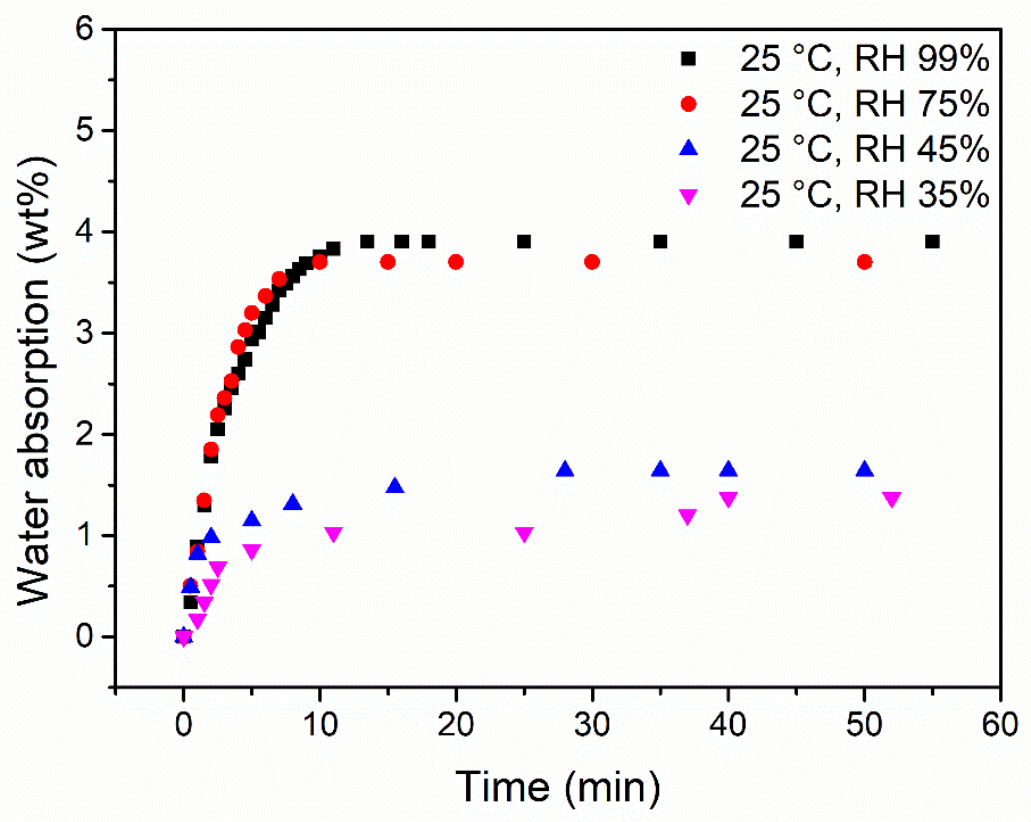

Figure S16. Water absorbance curves of II. 
4. X-Ray Diffraction (XRD) pattern of II-water adhesive materials

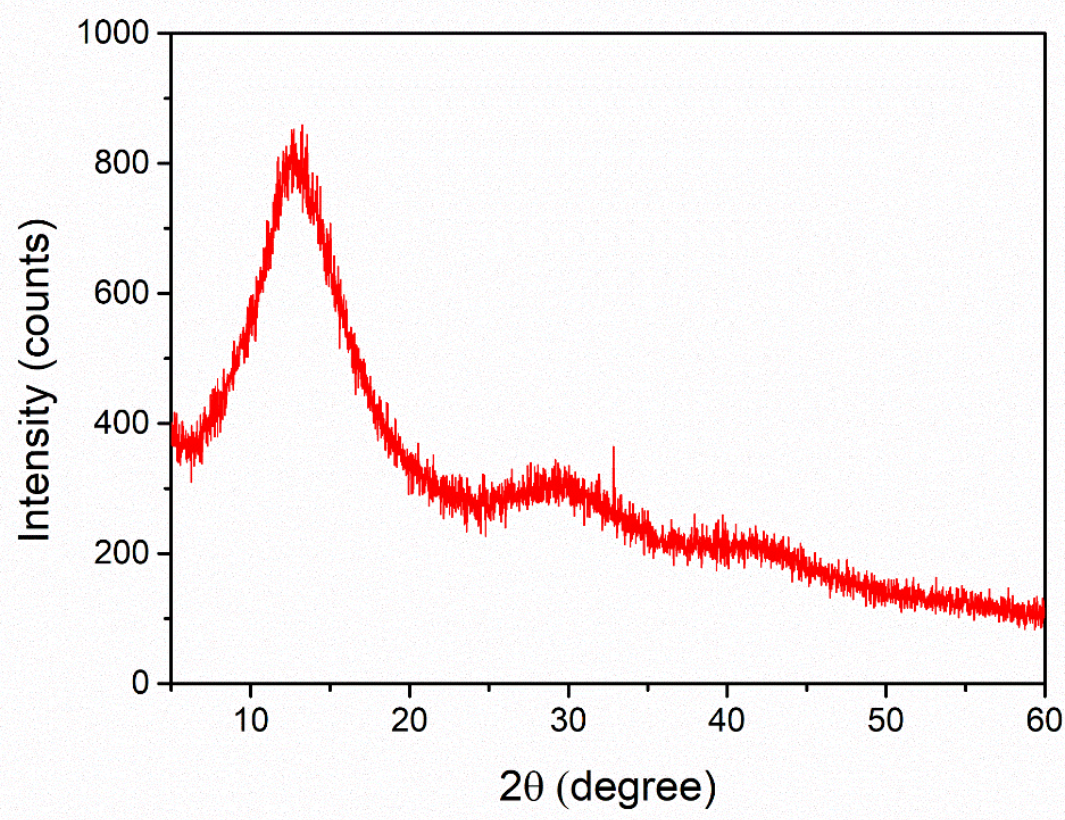

Figure S17. XRD pattern of II-water adhesive materials (sample was coated on a silicon surface, the water content is about $1.5 \mathrm{wt} \%$ ).

4. Rheology measurements of II-water adhesive materials

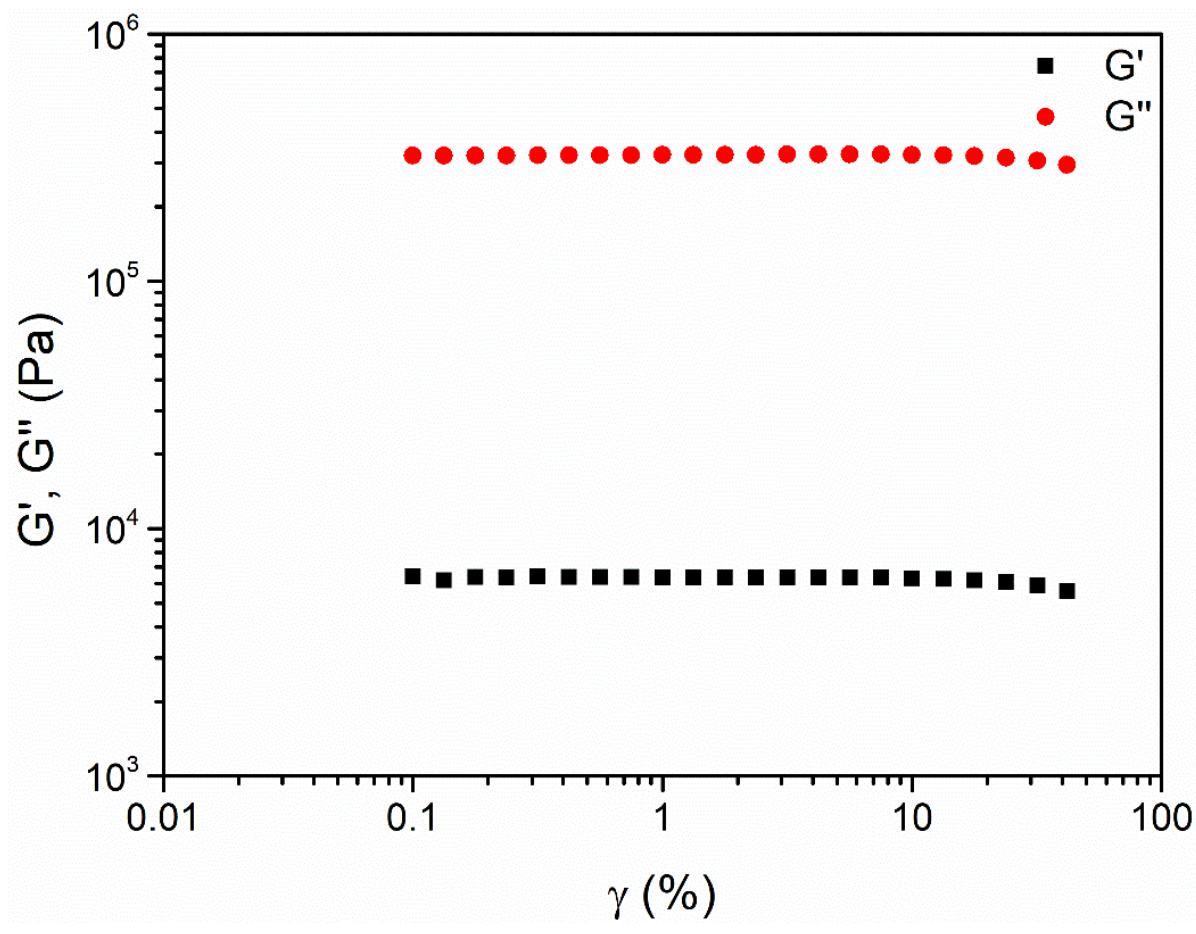


Figure S18. $G$ ' and $G$ ” values of II: $\mathrm{H}_{2} \mathrm{O}(100: 1.3$, wt $\%: w t \%)$ on strain sweep.

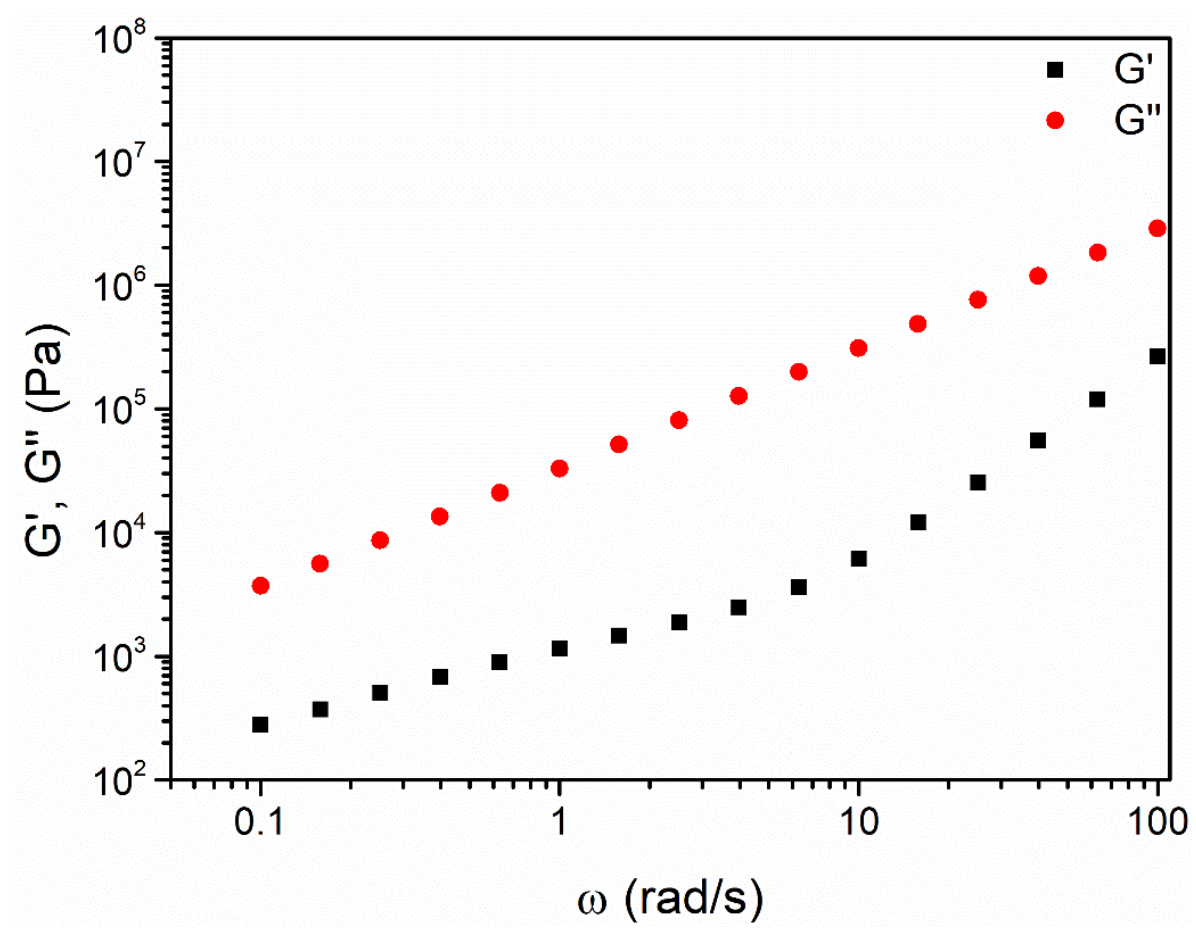

Figure S19. $G$ ' and $G$ "' values of II: $\mathrm{H}_{2} \mathrm{O}(100: 1.3$, wt $\%: w t \%)$ on frequency sweep.

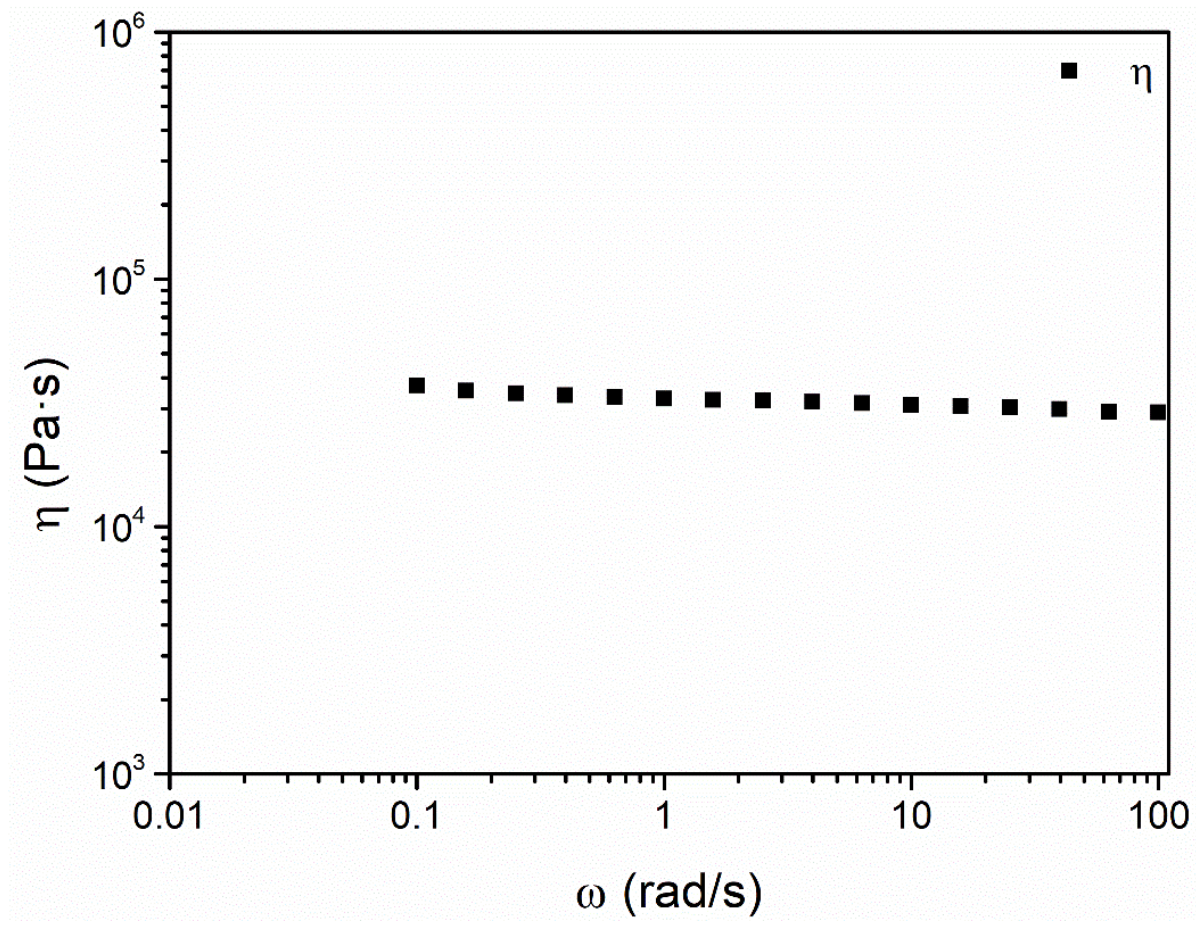

Figure S20. $\eta$ values of II: $\mathrm{H}_{2} \mathrm{O}(100: 1.3$, wt $\%: w t \%)$ on frequency sweep. 


\section{IR spectroscopy of II-H $\mathrm{H}_{2} \mathrm{O}$ and II-D $\mathrm{D}_{2} \mathrm{O}$ materials}

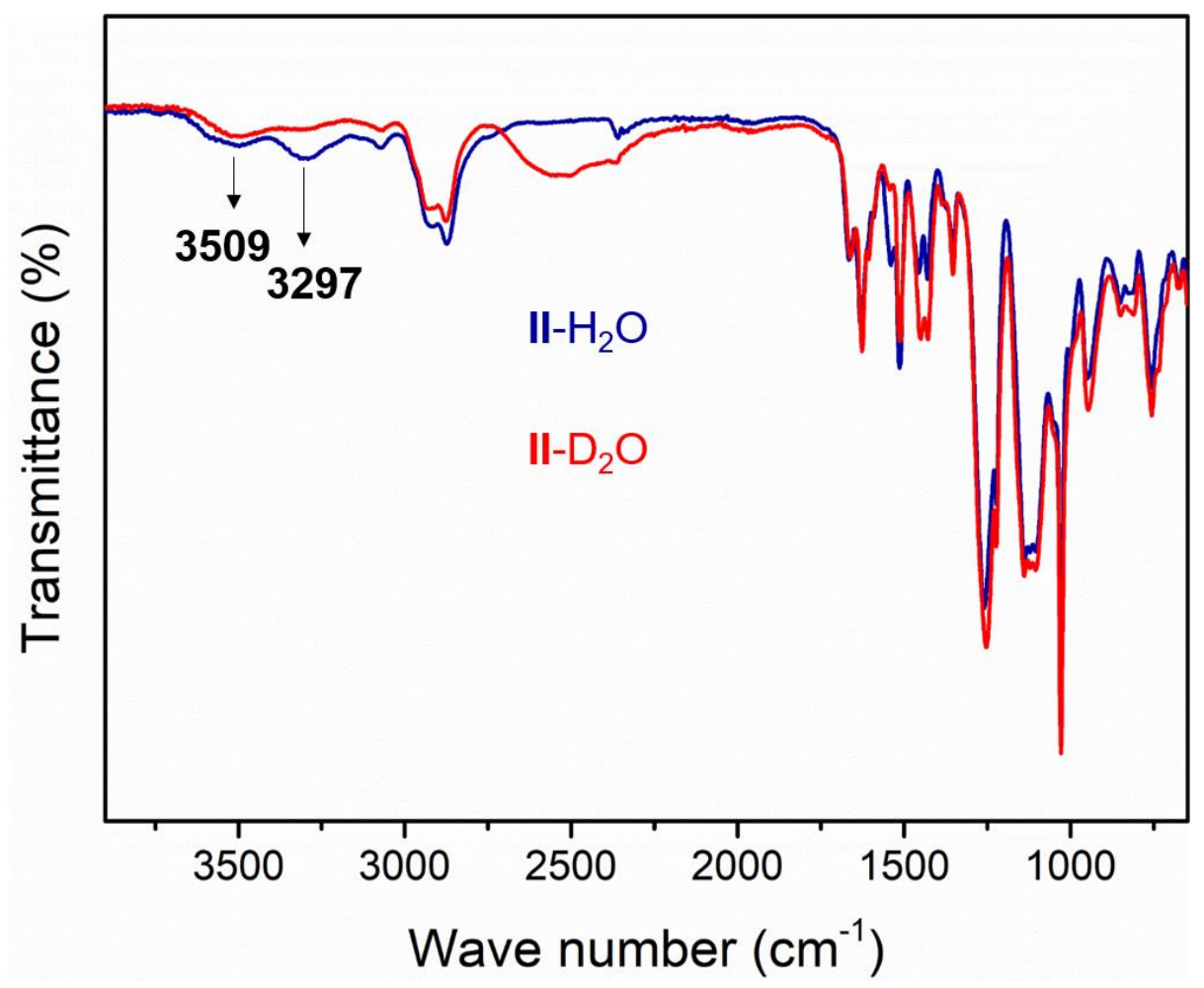

Figure S21. IR spectra of II- $\mathrm{H}_{2} \mathrm{O}$ and II- $\mathrm{D}_{2} \mathrm{O}$.

6. Thermogravimetric Analysis (TGA) characterization of II-water materials

(a)

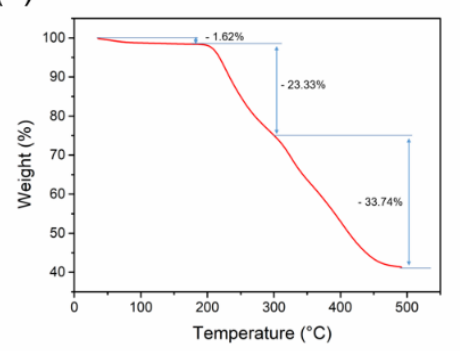

(b)

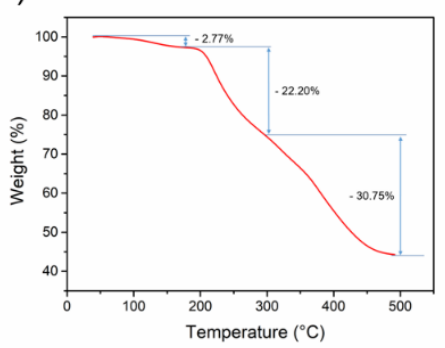

(c)

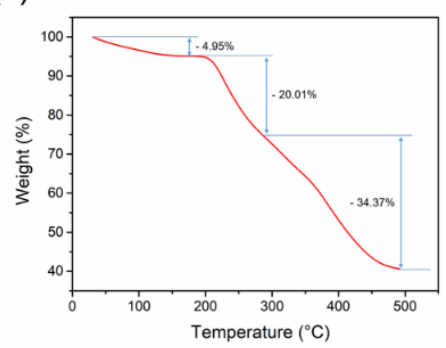

Figure S22. TGA measurements of II-water materials with different water contents. 


\section{Scanning electron microscopy (SEM) of the micromorphology of}

\section{II-water adhesive materials}

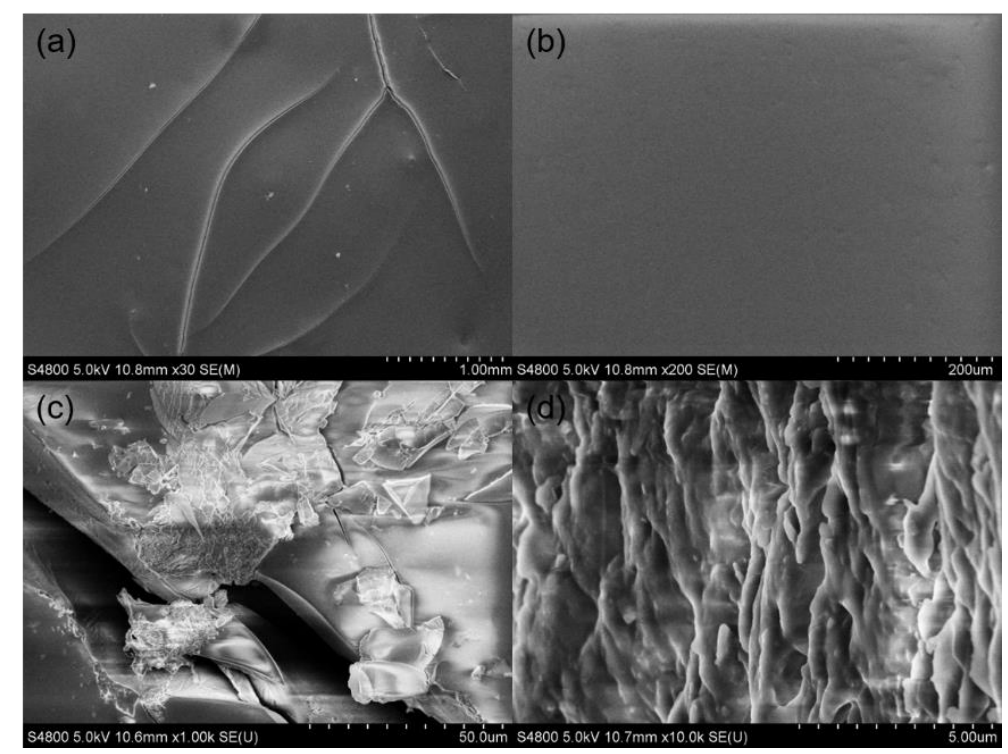

Figure S23. SEM images of II-water adhesive materials. From SEM images, we cannot find a detailed morphological structure of II.

\section{DFT calculation}

The density functional theory (DFT) calculations were performed using Gaussian 16, Revision B.01. ${ }^{\mathrm{S} 3}$ The geometry optimizations were conducted using the M06-2X functional, ${ }^{\mathrm{S} 4}$ with Lanl2dz basis set ${ }^{\mathrm{S} 5}$ for $\mathrm{Pt}$, and 6-31G(d) basis set for the other atoms. To confirm whether each optimized stationary point is an energy minimum, as well as evaluate the zero-point vibrational energy and thermal corrections at $298 \mathrm{~K}$, the vibrational frequencies were computed at the same level of theory as for the geometry optimizations. On the basis of the gas-phase optimized structures, the single-point energies were evaluated with the M06-2X functional, SDD basis set for Pt, and 6- 
$311+\mathrm{G}(\mathrm{d}, \mathrm{p})$ basis set for other atoms. Energies reported are free energies in $\mathrm{kcal} / \mathrm{mol}$. Geometries of the optimized structures shown were displayed with CYLView. ${ }^{\text {S6 }}$

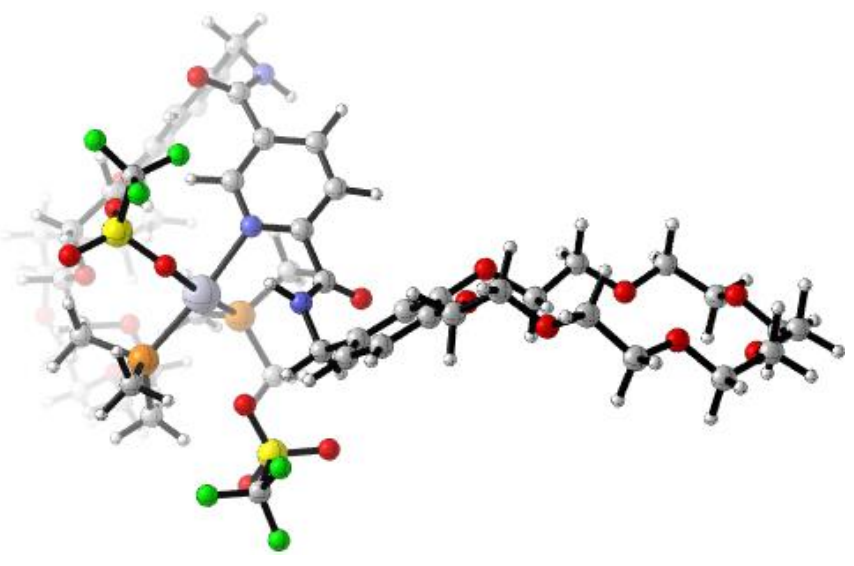

Figure S24. DFT calculation of II.
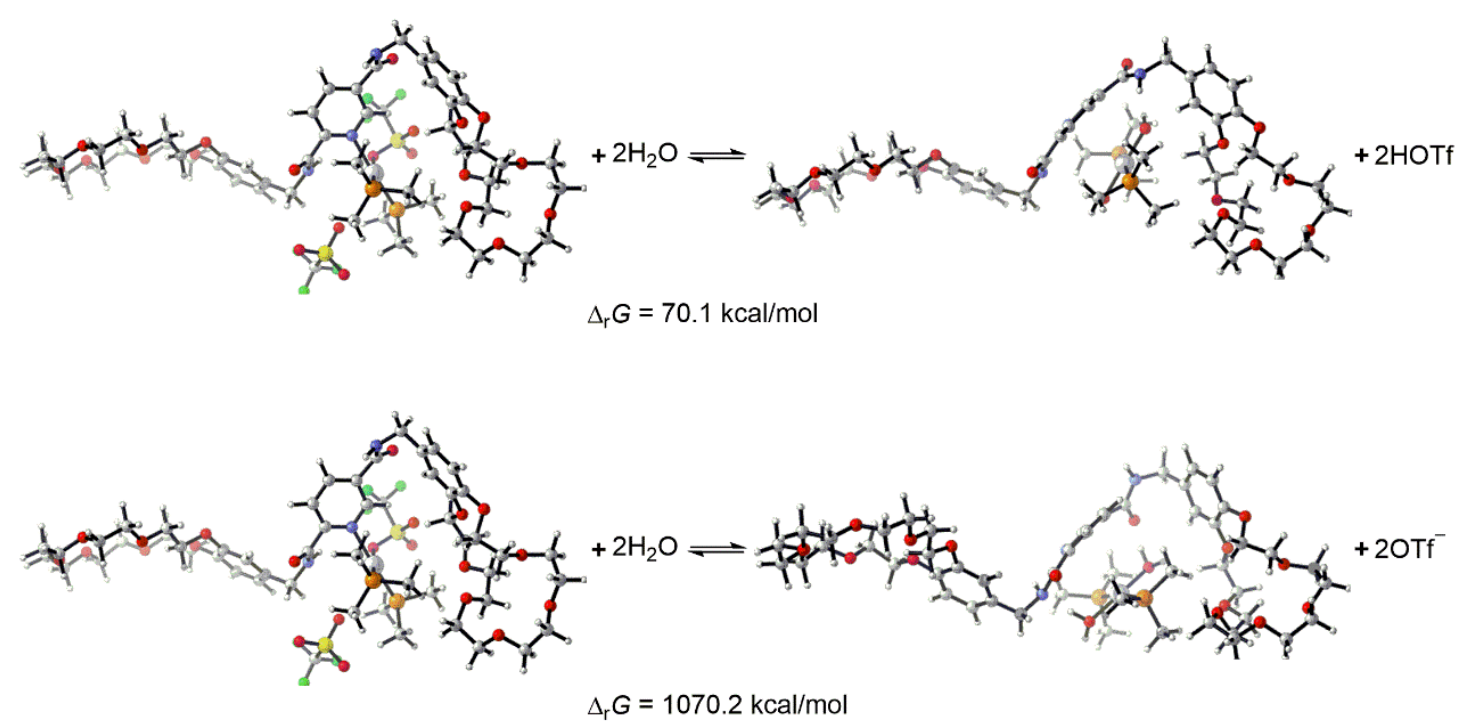

Figure S25. DFT calculation of II with water molecules.

\section{References}

(S1) Qi, Z. H.; de Molina, P. M.; Jiang, W; Wang, Q.; Nowosinski, K.; Schulz, A.; Gradzielskib, M.; Schalley, C. A. Chem. Sci. 2012, 3, 2073-2082.

(S2) Stang, P. J.; Cao, D. H.; Saito, S.; Arif, A. M. J. Am. Chem. Soc. 1995, 117, 62736283. 
(S3) Gaussian 16, Revision B.01, Frisch, M. J.; Trucks, G. W.; Schlegel, H. B.; Scuseria, G. E.; Robb, M. A.; Cheeseman, J. R.; Scalmani, G.; Barone, V.; Petersson, G. A.; Nakatsuji, H.; Li, X.; Caricato, M.; Marenich, A. V.; Bloino, J.; Janesko, B. G.; Gomperts, R.; Mennucci, B.; Hratchian, H. P.; Ortiz, J. V.; Izmaylov, A. F.; Sonnenberg, J. L.; Williams-Young, D.; Ding, F.; Lipparini, F.; Egidi, F.; Goings, J.; Peng, B.; Petrone, A.; Henderson, T.; Ranasinghe, D.; Zakrzewski, V. G.; Gao, J.; Rega, N.; Zheng, G.; Liang, W.; Hada, M.; Ehara, M.; Toyota, K.; Fukuda, R.; Hasegawa, J.; Ishida, M.; Nakajima, T.; Honda, Y.; Kitao, O.; Nakai, H.; Vreven, T.; Throssell, K.; Montgomery, J. A., Jr.; Peralta, J. E.; Ogliaro, F.; Bearpark, M. J.; Heyd, J. J.; Brothers, E. N.; Kudin, K. N.; Staroverov, V. N.; Keith, T. A.; Kobayashi, R.; Normand, J.; Raghavachari, K.; Rendell, A. P.; Burant, J. C.; Iyengar, S. S.; Tomasi, J.; Cossi, M.; Millam, J. M.; Klene, M.; Adamo, C.; Cammi, R.; Ochterski, J. W.; Martin, R. L.; Morokuma, K.; Farkas, O.; Foresman, J. B.; Fox, D. J. Gaussian, Inc., Wallingford CT, 2016.

(S4) (a) Zhao, Y.; Truhlar, D. G. Theor. Chem. Acc. 2008, 120, 215. (b) Zhao, Y.; Truhlar, D. G. Acc. Chem. Res. 2008, 41, 157.

(S5) (a) von Szentpaly, L.; Fuentealba, P.; Preuss, H.; Stoll, H. Chem. Phys. Lett. 1982, 93, 555. (b) Dolg, M.; Wedig, U.; Stoll, H.; Preuss, H. J. Chem. Phys. 1987, 86, 866. (c) Schwerdtfeger, P.; Dolg, M.; Schwarz, W. H. E.; Bowmaker, G. A.; Boyd, P. D. W. J. Chem. Phys. 1989, 91, 1762.

(S6) Legault, C. Y. CYLView, version 1.0b; Universitéde Sherbrooke: Sherbrooke, QC, Canada, 2009; http://www.cylview.org. 